\title{
¿Preeclampsia, o hipertensión simplemente? Una señal de alarma
}

\author{
Bernardo Agudelo Jaramillo, MD, GO.*
}

\begin{abstract}
RESUMEN: La expresión clínica de una alteración hipertensiva durante la gestación como la llamada "preeclampsia", refleja simplemente la incapacidad funcional del organismo materno para tolerar las adaptaciones fisiológicas de la gestación y/o las cargas que a ella se adicionen. Lógicamente el deterioro se considera funcional, o sea transitorio mientras persista la sobrecarga o la reserva. El tiempo requerido para que la enfermedad se manifieste es el tiempo reserva, y la reserva puede recuperarse al estado evolutivo de la línea normal de declinación, pero muy probablemente más disminuida. Cuando ya se han consumido todos los elementos de tolerancia a la sobrecarga funcional, cualquiera que ella sea (evento fisiológico gestacional, noxa, sobrecarga, etc.) aparece la enfermedad clínicamente manifiesta y se declara la Hipertensión Arterial Crónica. En este momento se hace evidente el deterioro orgánico con las consecuencias de la enfermedad y el camino hacia la muerte, innegable proceso al que todo ser vivo se ve condenado, pero que en definitiva se acelera con la presencia de una enfermedad.
\end{abstract}

PALABRAS CLAVES: Hipertensión, embarazo, preeclampsia.

SUMMARY: The clinical expression of a hypertensive alteration during pregnancy like the one called preeclampsia, reflects the functional incapacity of the maternal organism, for tolerating the fisiologic adaptationsof pregnancy and/or the additional freights. The deterioration is considered as functional; it is transitory while an overchargeor reservation persists. The required time for the sickness revelation is the reservation time, and the reservation can be regained at the evolutional state of declination, but probably more decreased. When the tolerating elements have already consumed to the functional overcharge (fisiologic events, noxa, overcharge, etc.) the manifested clinical sickness appears and the Chronical Arterial Hypertension declares. At this moment, the organic deterioration reveals with the sickness consequences and the path to death.

KEY WORD: Hypertension, pregnancy, preeclampsia.

\section{Introducción}

La prevalencia de la enfermedad hipertensiva es del $20 \%$ (1-2) y varía según factores de grupo (étnicos, raciales, sexo, culturales, etc.). El diagnóstico temprano logra disminuir la morbimortalidad dependiente de enfermedad coronaria, enfermedad arteroesclerótica y accidente cerebrovascular, que son las principales causas de limitación física en la tercera edad. Los hombres de 40-59 años con presión diastólica entre 90-114 tienen un exceso de muerte entre 5-12.5\%; la enfermedad coronaria en la mujer es tres veces superior cuando la presión arterial alcanza 160/95 y en los hombres es dos y media veces mayor; el accidente trombótico es 7-8 veces superior y la falla cardíaca 5-7 veces. Los factores de riesgo guían hacia el diagnóstico precoz y en consecuencia mejoran la prevalencia, disminuyen la morbilidad y consecuentemente la mortalidad. De esta forma, la enfermedad hipertensiva se considera un problema de Salud Pública que exige esfuerzos interdisciplinarios con el fin de abordarla racionalmente.

* Profesor del Departamento de Ginecología y Obstetricia. Programa de Reproducción. Facultad de Medicina. Universidad de Antioquia.

Ganancia de Retroalimentación, es una forma de cuantificar la capacidad de un sistema para mantener estable la variable, es una forma de medir la reserva.
Consideramos la gestación un experimento biológico, en el que la mujer se somete a multitud de variaciones de su ambiente interno para facilitar el desarrollo del producto gestacional. Estas variaciones ocurren en tan sólo 280 días, plazo relativamente corto comparado con la evolución de otras patologías, que no alcanzan a desarrollar tantas modificaciones simultáneamente. Se pone en evidencia la capacidad adaptativa de la naturaleza femenina para tolerar la gestación "normal" y se resalta su potencial de riesgo para desencadenar eventos patológicos o alterar otros ya presentes.

El trastorno hipertensivo, es la patología más frecuentemente asociada a la gestación (5-15\%) e igualmente se acompaña de elevados índices de morbimortalidad (3-4). La incidencia depende de condiciones poblacionales y factores de riesgo similares a la hipertensión esencial (obesidad, raza negra, antecedentes metabólicos, familiares entre otros). El $50 \%$ de estas pacientes desarrollan posteriormente hipertensión crónica (5). Uno de los problemas principales es la variedad de formas clínicas que adopta y que ha dado origen a diferentes clasificaciones, de las cuales la más aceptada es la de la Asociación Americana de Obstetricia y Ginecología: $(3,19)$.

- Hipertensión crónica. - Preeclampsia-Eclampsia.

- Preeclampsia sobreagregada a Hipertensión crónica.

- Hipertensión transitoria - No clasificada. 
Figura 1

COMPORTAMIENTO DE LAS PRESIONES

SISTOLICA Y DIASTOLICA DURANTE LA

GESTACION NORMAL EN NULIPARAS Y

MULTIPARAS

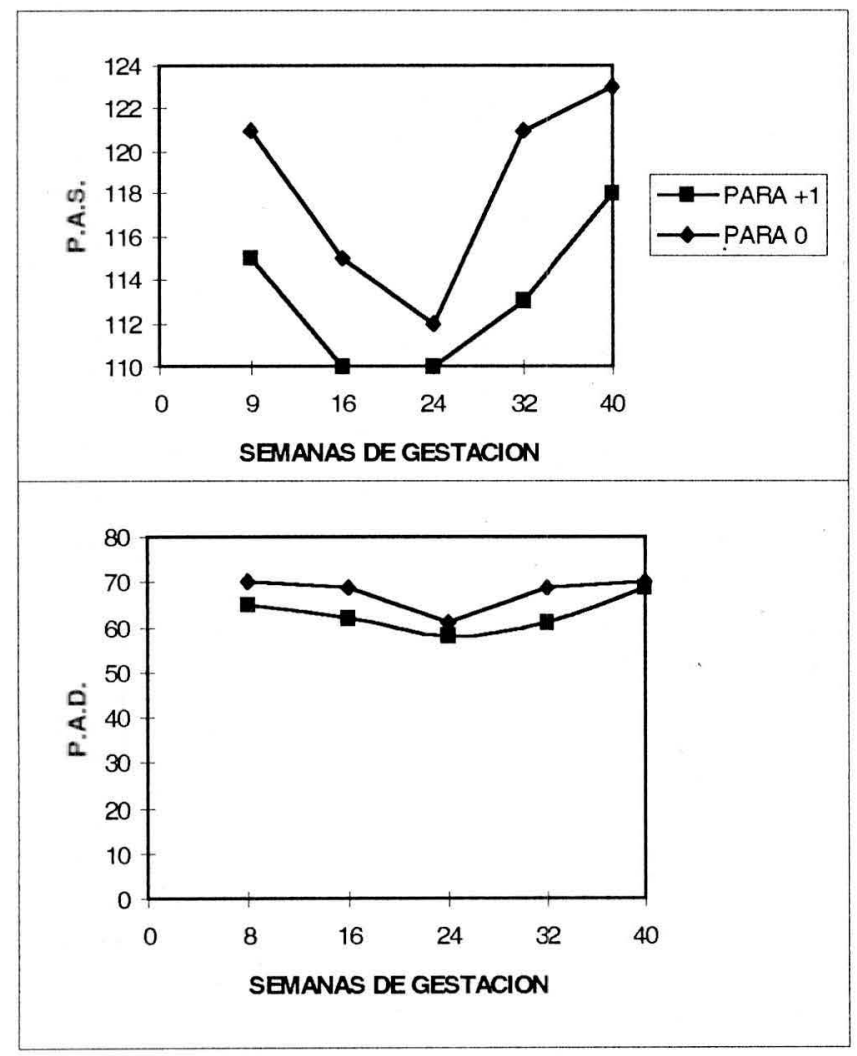

Los límites normales de presión (igual o menor de 140/90) deben analizarse con cautela en la mujer, teniendo en cuenta que en ella la presión arterial fluctúa entre 100-110/60-80 y principalmente en la gestación, en la que se espera una reducción fisiológica del $25 \%$ en las cifras tensionales (fig. 1). De esta forma, una presión media en el segundo trimestre mayor a $90 \mathbf{~ m m ~ d e ~} \mathbf{H g}$ (34) se considera anormal.

El comportamiento anormal de la presión arterial se asocia a elevación de la morbilidad fetal $(3,6)$, en la mujer a mayor morbilidad cardiovascular y elevado riesgo de deterioro en la calidad de vida a largo plazo. Por lo tanto se requiere la detección temprana en la gestación para garantizar medidas preventivas oportunas.

\section{Consideraciones fisiopatológicas}

La presión arterial es una medida del trabajo cardíaco como bomba, para suministrar la sangre a los tejidos en un sistema cerrado de circulación, condicionada por dos factores.

$$
\text { P.A }=\text { G.C } \times \text { R.P }
$$

De donde G.C es el gasto cardíaco y R.P la resistencia vascular periférica. El gasto cardíaco depende del volumen y de la frecuencia.

$$
\mathbf{G . C}=\mathbf{V} \times \mathbf{F}
$$

Figura 2

\section{MECANISMOS REGULADORES DE LA PRESION ARTERIAL Y GANANCIAS DE RETROALIMENTACION}

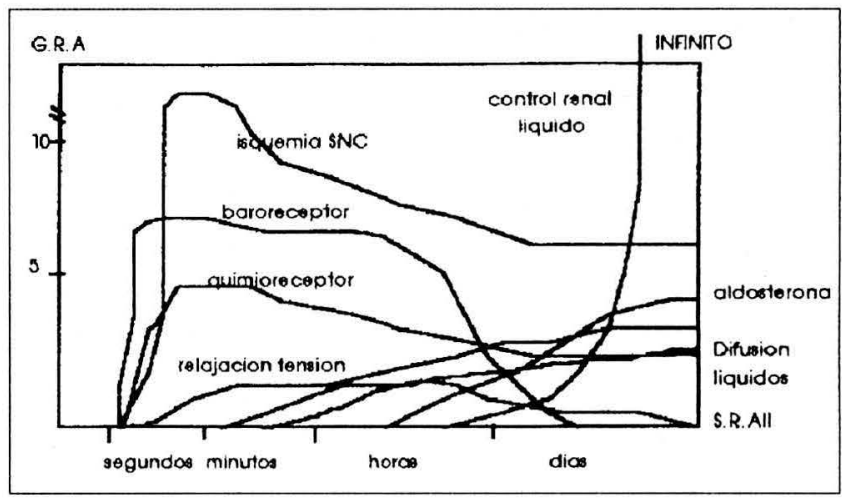

Reemplazando, la presión arterial es modificada por el volumen, la frecuencia y la resistencia arterial, así:

$$
\text { P.A }=\text { V } \times \text { F } \times \text { R.P }
$$

La presión arterial tiende a elevarse en el curso de los años con acentuación hacia la quinta década a pesar de la existencia de mecanismos fisiológicos reguladores, que en conjunto constituyen la "reserva individual". La reserva es determinada genéticamente y se modifica en el curso de la vida por su deterioro natural y/o por la aparición de condiciones deletéreas que consumen los reguladores existentes (factores de riesgo). Cuando aparece la enfermedad, la "reserva" alcanza un "punto crítico" en el cual se rompe el equilibrio entre la salud y los precipitantes $(1-2,7)$.

La presión arterial se conserva en un estrecho rango de "normalidad" gracias a mecanismos reguladores que intervienen constantemente y en forma simultánea, pero con predominio de unos a otros según el tiempo de acción y con diferentes ganancias de retroalimentación (G.R.A) ${ }^{1}$ $(5,8)$, ellos son:

Control inmediato, comprende el sistema de baroreceptores que actúa reflejamente a través del sistema nervioso autónomo (S.N.A) y conserva la presión dentro de un rango estrecho de 60 a $180 \mathrm{~mm}$ de $\mathrm{Hg}$ : el sistema de quimioreceptores que por estímulo químico conserva los niveles de perfusión, principalmente de órganos vitales y el sistema de respuesta isquémica del sistema nervioso central, que funciona en un estrecho límite de presión y facilita respuestas reflejas hipertensivas o hipotensivas. Tienen una ganancia de retroalimentación pequeña y por ende poco valor para conservar la presión arterial estable a largo plazo, sufren un proceso de adaptación. Facilitan e interactúan con los factores intermedios.

Control intermedio, comprende el sistema ReninaAngiotensina-Aldosterona (S.R-AII-A) en respuesta a cambios de perfusión renal, modificaciones osmóticas y otros factores infrarenales, origina vasoconstricción (angiotensina II) y retención de sodio y agua (aldosterona); los sistemas de autorelajación vascular y de difusión de líquidos a los espacios. Este tipo de control posee una 
G.R.A baja, de poca importancia en la regulación a largo plazo pero valiosa como punto medio entre el control del S.N.A simpático y los mecanismos de acción prolongada, debido a la estimulación de la actividad de renina por el simpático y sensibilización a la vasoconstricción de la A-II.

Además, la aldosterona se conecta al mecanismo de acción a largo plazo.

Control a largo plazo, es el más importante por su casi infinita G.R.A y su perdurabilidad, con limitada autorregulación en respuesta a situaciones de crisis. Comprende el sistema de autorregulación renal de líquidos y la acción de la aldosterona con los cuales conserva en un estrecho de presión, el balance de egreso urinario con el ingreso diario, de donde una elevación de presión de tan sólo $6 \mathrm{~mm}$ de $\mathrm{Hg}$ aumenta siete veces la diuresis normal (curva A). A su vez, si aumenta la ingesta líquida, se

Figura 3

AUTOREGULACION RENAL DE LIQUIDOS Y SU RELACION CON LA PRESION ARTERIAL

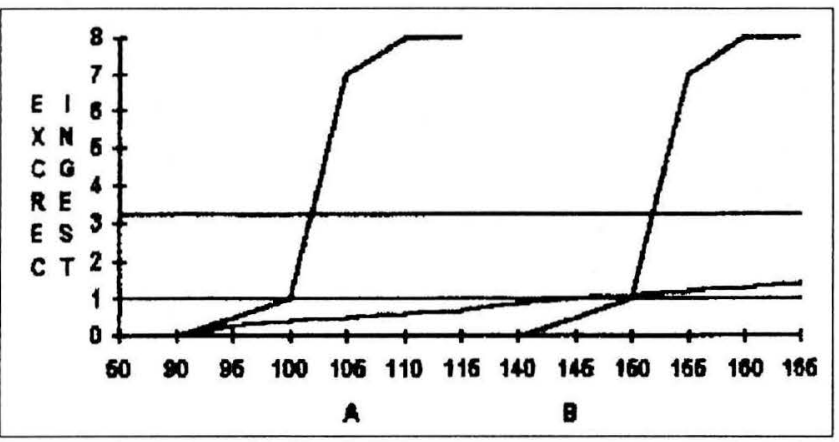

necesita elevar la presión de perfusión desplazando la curva hacia la derecha (curva B) para regular el volumen plasmático (fig. 3). La elevada G.R.A habla por sí misma de la reserva, responsable de mantener la presión arterial en un rango de normalidad a pesar de pérdidas renales hasta del $70 \%(5,8)$.

El complejo control de la perfusión depende del balance entre sustancias vasoconstrictoras y vasodilatadoras (9) y de su íntima relación con los mecanismos de regulación, ej: la angiotensina II sistémicamente causa vasoconstricción, en forma autocrina sensibiliza la célula vascular al efecto de la norepinefrina, a nivel renal estimula la síntesis de prostaciclina y su acción vasopresora desencadena en el endotelio la síntesis de péptidos vasodilatadores (péptido endotelial vasodilatador, sistema kinina-kalikreína, etc). (1).

Todos los mecanismos autorreguladores locales son determinados genéticamente; el más demostrado es el sistema enzimático kininógeno-kinina-kalikreína que libera del endotelio el péptido endotelial vasodilatador, facilita la síntesis de prostaglandinas $\left(\mathbf{P g I}\right.$ y $\left.\mathbf{P g} \mathbf{E}_{2}\right)$, relaja el músculo liso, a nivel renal libera renina, además la enzima convertidora de la angiotensina I es una kinina. En hipertensos crónicos y en sus descendientes se encuentra disminución de la producción de kininas-kalicreínas; otros investigadores demuestran una disminución de la kalikreína urinaria en niños normotensos hijos de madres hipertensas $(1,9)$ y en preeclámpticas $(10)$.
El óxido nítrico, que parece ser el péptido endotelial vasodilatador, participa activamente en la fisiopatología de la hipertensión, es liberado por las kininas a través del $\mathrm{GMP}_{\mathrm{c}}$ y se cree que sea el mediador de los efectos vasodilatadores del endotelio (10-11, 41).

Otros factores son: el calcio, como mediador de la secreción y respuesta de algunas hormonas y compuestos vasoactivos, modulador de la contracción muscular lisa (1), posible utilidad preventiva y coadyuvante terapéutica en hipertensión (12), además de un papel predictivo cuando se evalúa la excreción renal $(6,13)$ y en el citoplasma de plaquetas (14); el potasio, modulador del simpático y con efecto natriurético, función determinada genéticamente (15), cuando aumenta se disminuye la presión arterial y facilita la terapia antihipertensiva; la bomba del sodio/potasio regula los gradientes iónicos transmembrana y se evidencia una disminución de su actividad en poblaciones de alto riesgo de hipertensión (negros, hombres) (1), a este nivel confluyen las acciones del S.R.A-II.A, corticosteröides y los diferentes iones, entre otras, donde se ejecuta el tono autónomo dominante. Otros iones interactúan con antagonistas, como el magnesio con el calcio y el sodio con el potasio. Se detectan polipéptidos que regulan el tono, bien directamente (factor inhibidor de la bomba de sodio-potasio, endotelina) o indirectamente modificando el volumen (Factor natriurético auricular) $(1,9,16-17)$.

En resumen, el tono vasomotor resulta de la compleja interacción entre el S.N autónomo y los mecanismos periféricos de autorregulación, que reúnen la actividad neural con los procesos químicos. El endotelio vascular es considerado una unidad funcional donde se procesan todos los estímulos y de donde surge la respuesta final: el Tono. De esta manera, el comportamiento de la presión arterial depende de los factores de Reserva reunidos a nivel del endotelio vascular y es aquí donde se encuentra la razón por la cual un individuo desarrolla hipertensión y otro no $(9-11,18)$. Los elementos de respuesta vascular se consumen en el tiempo, situación que favorece el deterioro normal o envejecimiento. El proceso puede acelerarse si se agregan facilitadores (factores de riesgo) que se potencializan en sus efectos deletéreos, con progresivo deterioro de la reserva y en consecuencia mayor morbilidad y mortalidad (fig. 4).

Figura 4

CONCEPCION DE LA RELACION ENTRE LA RESERVA FUNCIONAL, EL TIEMPO RESERVA Y LA APARICION DE LOS FACTORES DE RIESGO CON RESPECTO A LA MORBILIDAD Y MORTALIDAD HIPERTENSIVA

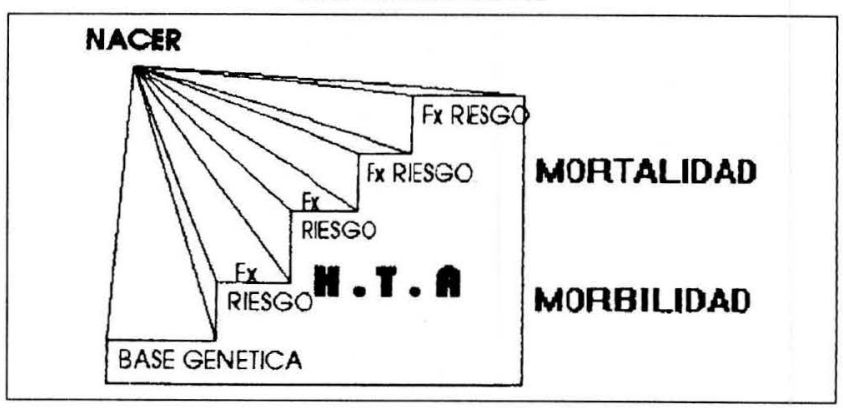


El tiempo que transcurre entre la normalidad (reserva intacta) y la aparición de la enfermedad (punto crítico), lo llamamos tiempo reserva. Esta nueva definición nos aproximará fácilmente hacia la comprensión de la hipertensión en la gestación.

Se plantean para la hipertensión arterial tres interesantes hipótesis:

1 La hipertensión arterial es una enfermedad endotelial.

2. Tiene una herencia multifactorial, y

3. Es un proceso de envejecimiento por la endoperoxidación de los ácidos grasos de las membranas.

\section{Fisiopatología de la hipertensión en la gestación}

Las modificaciones hemodinámicas de la gestación generan una "tendencia hipertensiva" con la cual se pretende facilitar el desarrollo del producto gestacional. La adaptación a esa situación requiere normalidad fisiológ $i$ ca, es decir, un conjunto óptimo de: a) elementos de regulación vascular genéticamente determinados, b) integridad de los mecanismos de autoregulación dependientes de la condición de salud individual y c) de condicionantes externos, propios del ambiente (tensión familiar, laboral, social, etc), los que influyen actuando sinérgicamente para conservar el "tono normal".

La gestación es considerada un alloinjerto cuya tolerancia depende de la respuesta inmune materna a antígenos paternos, sin alterar la unidad fetoplacentaria. Son controvertidas las opiniones acerca de si el fenómeno depende de un bloqueo inmunitario o de una activación específica protectora, sin embargo en lo que respecta a su compromiso en la hipertensión, la placenta parece ser el órgano de choque inmune, por lo menos en la forma preeclámptica. Soportando el factor inmune, se propone que: si ambos padres son antigénicamente similares o ha ocurrido un proceso previo de sensibilización antigénica (exposición coital prolongada), la hipertensión en la gestación es menos frecuente; existe relación directa entre la frecuencia del trastorno hipertensivo y la masa placentaria antigénica (gemelar, mola); otras asociaciones inmunológicas como la respuesta inmune similar entre el riñón y la placenta, la aparición de hipertensión cuando se daña la unión coriodecidual por mecanismos inmunes experimentales, la predominancia en la primera gestación, etc. $(3,7,19-20)$. Sin menospreciar la importante influencia inmunológica que puede explicar muchas situaciones de hipertensión en la gestación, no consideraremos este proceso sino desde el punto de vista de la sobrecarga funcional que soportan órganos vitales de la economía materna, como arterias deciduales, el riñón, la placenta y el hígado, que siendo órganos blancos para la actividad inmune, sufren un proceso de deterioro en su reserva funcional.

Brosens en 1972 (21) describe la invasión del sincisiotrofoblasto, que comienza alrededor de la semana 8 y va hasta la semana $16-20$, donde el endotelio y la íntima vascular de las arterias deciduales y aún de las espirales miometriales, son reemplazadas por una rígida pared trofoblástica que baja la resistencia de perfusión. En las pacientes que desarrollan hipertensión, la invasión no ocurre adecuadamente, al parecer determinado por factores inmunes y por el componente genético del producto (fig. 5). Las modificaciones gestacionales dependen directa e indirectamente del efecto de las hormonas esteroideas, estrógenos y progesterona, que inducen procesos con tendencia hipertensiva y a su vez reguladores fisiológicos, así:

Figura 5

REPRESENTACION DE LAS ARTERIAS ENDOMETRIALES Y MIOMETRIALES DURANTE LA GESTACION NORMAL Y EN LA PREECLAMPSIA COMO CONSECUENCIA DE LA INVASION DEL SINCISIOTROFOBLASTO (BROSENS, 1972)

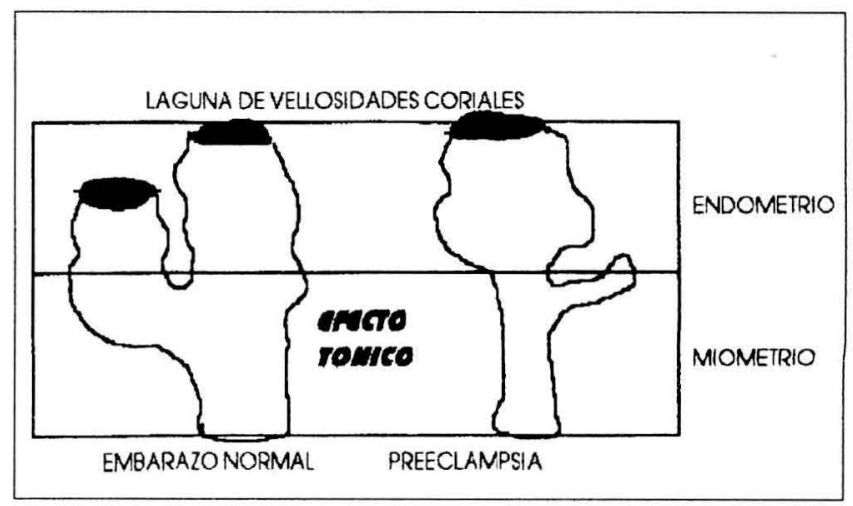

Estrógenos, activan el sistema R.A-II.A por inducción de síntesis hepática de angiotensinógeno y substrato de renina, inducen el kininógeno (elevación de kininas), favorece la síntesis placentaria de prostaciclina $\left(\mathrm{PG}-\mathrm{I}_{2}\right)$ y $\mathrm{PG}-\mathrm{E}_{2}$ (vasodilatación local) y facilita la síntesis endotelial del óxido nítrico, entre otras (22-23).

Progesterona, sirve como precursor de los estrógenos, posee efecto natriurético directo (antialdosterónico), a nivel placentario inhibe la síntesis de prostaciclina, se metaboliza por dos vías: a través de la 21-hidroxilasa produce Doca, precursor de la aldosterona y a través de la 5 alfa-reductasa produce 5 alfa-dihidro-progesterona, que compite con los receptores de angiotensina II (tolerancia vascular al efecto de los vasoconstrictores) (1, 3, 23-26).

El efecto conjunto de estas dos hormonas eleva el S.R.A-II.A y por lo tanto genera un hiperaldosteronismo secundario, con retención de agua y sodio (aumento del 40-50\% del volumen plasmático y retención de 500-900 $\mathrm{m} \mathrm{Eq} \pm$ de sodio) (7), con el fin de satisfacer las demandas hemodinámicas de la gestación. La progesterona podría ser desencadenante del aldosteronismo o por el contrario, ser una consecuencia compensatoria al hiperaldosteronismo por su conocida acción competitiva y perdedora de sal. El embarazo que cursa con hipertensión presenta depresión del S.R.A-II.A, quizás en respuesta a la excesiva retención de sodio por el deterioro de la función renal, además, cuando aumenta el tono vasomotor se eleva a nivel renal la retención de sodio con el fin de llenar el espacio vascular $(1,7,23,27)$.

El efecto aldosterónico aumenta el volumen circulante con el cual se eleva la presión de perfusión, en su activación se expone el organismo materno a la 
angiotensina II que es el más potente vasoconstrictor conocido. Hasta este momento el embarazo muestra una tendencia hipertensiva, pero los procesos adaptativos generan una condición de Resistencia vascular a la angiotensina II, que caracteriza la evolución normal del embarazo. Esta resistencia es mediada por:

- La autoregulación de receptores de la angiotensina II (regulación negativa).

- El balance de producción con las bradikininas (vasodilatadoras) que a su vez estímulan la síntesis de $P G^{\prime} I$, y la $P G^{\prime} E$, ambas vasodilatadoras.

- Balance esntre las prostaglandinas vasodilatadoras y las vasoconstrictoras, existiendo en la gestación normal una desviación hacia las vasodilatadoras.

- Efectos vasodilatadores e hipotensores fisiológicos de las hormonas esteroideas.

Gant en 1974, muestra que la resistencia vascular obedece a una "refractariedad" a la angiotensina, en la que se hace necesaria una dosis de angiotensina II de 8 $\mathrm{ng} / \mathrm{Kg} / \mathrm{min}$ para elevar la presión sistólica $20 \mathrm{~mm}$ de $\mathrm{Hg}$, condición que diferencia las pacientes con labilidad hipertensiva de aquellas con evolución normal (27). En las gestantes con hipertension arterial, la dosis de angiotensina es menor del valor umbral (fig. 6).

Figura 6

NIVELES DE ANGIOTENSINA II NECESARIOS PARA INDUCIR ELEVACION DE LA PRESION ARTERIAL, ENTRE EMBARAZADAS NORMALES Y LAS QUE DESARROLLAN PREECLAMPSIA EN EL CURSO DE LA GESTACION (GANT, 1974)

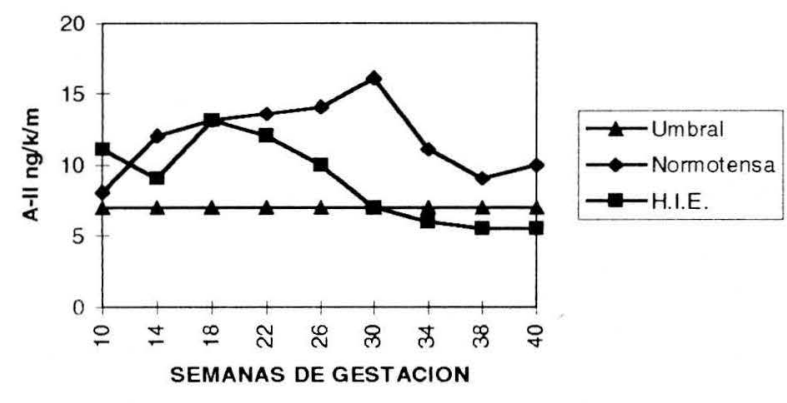

El deterioro en la síntesis de prostaglandinas vasodilatadoras que modulan la respuesta vascular en la gestación, se pone en evidencia durante los trastornos hipertensivos, así:

- Alteración en la biosíntesis de prostaglandinas de la serie n-3, derivadas del ácido linolénico (28).

- Disminución en la síntesis de prostaciclina (PG-I ${ }_{2}$ ) (29-32).

- Alteración del equilibrio entre PG- $\mathrm{I}_{2}$ y el TX-A, con pérdida de los efectos benéficos de la $\mathrm{PG}-\mathrm{I}_{2}$ como vasodilatadora, antiagregante plaquetario y citoprotectora endotelial (31).

La síntesis de prostaglandinas es constante y espontánea, al parecer como respuesta citoprotectora a las noxas que alteran la integridad de la membrana celular. En el endotelio normal, la $\mathrm{Pg}-\mathrm{E}_{2}$ es la principal prostaglandina sintetizada y no la $\mathrm{Pg} \mathrm{I}_{2}$, puesto que ésta última parece producirse exclusivamente cuando hay lesión vascular con activación de las plaquetas (33). Podría deducirse de estas afirmaciones, que las prostaglandinas No contribuyen en la regulación de la resistencia vascular periférica sino en condiciones patológicas (18), pero si se tiene en cuenta la intensa actividad endotelial de la unidad placentaria y su "deterioro" potencial al ser invadido por el trofoblasto invasor, parece lógico concluir que la elevada síntesis de $\mathrm{Pg} \mathrm{I}_{2}$ durante la gestación sea reflejo de la respuesta normal adaptativa en el lecho de contacto materno fetal.

La oxidación lipídica por medio de los radicales libres (peróxidos, superóxidos) hace parte de los procesos naturales de envejecimiento y enfermedad, durante los cuales se altera el microambiente celular. La relativa armonía entre los radicales libres de $\mathbf{O H}, \mathbf{O}$ y el complejo quelado de $\mathbf{F e}-\mathrm{O}_{2}$ y los antioxidantes naturales como las vitaminas E, A, C, la ceruloplasmina, entre otros, garantizan la conservación de una homeostasis relativamente normal, o esperada.

Cuando aparece la enfermedad hipertensiva, ocurre un disbalance conocido como "estrés oxidativo", capaz de bloquear el factor endotelial relajante, inducir espasmo vascular, bloquear el efecto de los antioxidantes naturales, inhibir la sintetasa de prostaciclina, inducir cambios en el endotelio vascular con liberación de enzimas lisosomales, TX-A, factor de crecimiento plaquetario, serotonina (34). A su vez, la biosíntesis de prostaglandinas es un proceso de peroxidación mediado por la lipo-oxigenasa y la ciclooxigenasa $(1,34,36)$.

En las pacientes con hipertensión durante la gestación ocurre una activación dg la peroxidación evidenciada por: (6, 34-35).

- Aumento del malondialdehído (metabolito medible de lípidos peroxidados).

- Elevación de los conjugados di-ene de los lípidos (40\% superior al valor normal en la semana 36 , doble de lo normal).

- Acumulación de pigmentos como la lipofuscina a nivel placentario y de lípidos insaturados, índice de activación de la peroxidación.

- Disminución de la biosíntesis de prostaciclina (PG-I, y activación de la agregación plaquetaria (aumento de TX-A, .

- Aumento de la fibronectina.

De esta información se concluye que la evolución del trastorno hipertensivo durante la gestación, sería la consecuencia final del desequilibrio entre los mecanismos reguladores vasculares determinados genéticamente y las modificaciones fisiológicas del ambiente interno (1). Cuando se rompe este frágil equilibrio, aparece la descompensación hemodinámica con la hipertensión como expresión de la enfermedad.

Como se ha esbozado, las adaptaciones hemodinámicas ocurren en respuesta a las elevaciones hormonales: aumento del volumen plasmático, del gasto cardíaco y de la frecuencia cardíaca. A pesar de estas modificaciones sobreviene un estado hipotensivo, con caídas de la presión arterial hasta del $25 \%$ del valor preembarazo, por lo cual Page y Christianson (37) sugieren que serviría como índice pronóstico de hipertensión. 
En la semana 8, Capeless (38) demuestra que ya hay cambios hemodinámicos importantes como, aumento de más del 50\% del gasto cardíaco alcanzando al final de la gestación (fig. 7) y descenso de la resistencia vascular periférica hasta un $30 \%$ del valor preconcepcional, entre otros.

Figura 7

MODIFICACIONES DEL GASTO CARDIACO Y DE LA RESISTENCIA PERIFERICA EN EMBARAZADAS NORMALES

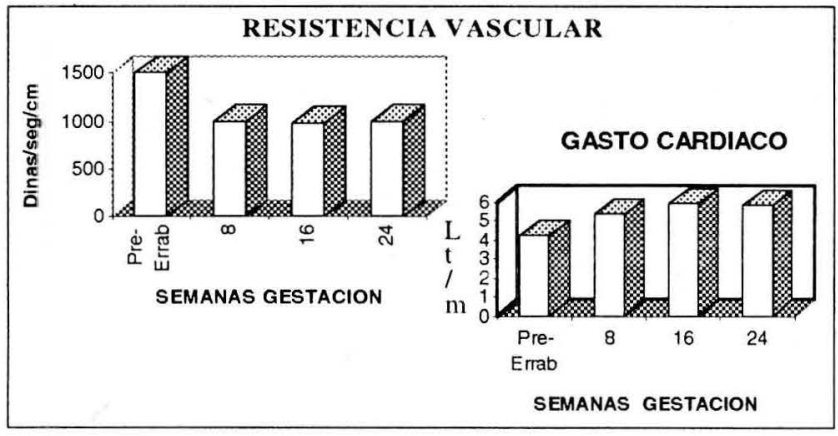

Propongo que para realizar un diagnóstico temprano de los trastornos hipertensivos, se tome toda la información que aporta la historia clínica, se evalúen muy conscientemente los signos hemodinámicos y se analicen integrados al comportamientode los cambios gestacionales esperados. Con mucha sutileza clínica y un gran sentido crítico es posible acercarse al diagnóstico temprano, siendo preferible equivocarse al decir "puede" desarrollar una hipertensión que tener que decir "ya tiene una hipertensión". Para lograr este objetivo y poder asumir conductas preventivas, se deben utilizar algunos hallazgos epidemiológicos como:

El riesgo de hipertensión aumenta notablemente cuando la madre o una hermana de la paciente ha tenido la enfermedad (22\% y $38 \%$ respectivamente) (7-39).

- La hipertensión establecida se eleva paulatinamente con el transcurrir de los años en mujeres que han tenido eclampsia en el embarazo (39) y aún formas leves de la llamada preeclampsia.

- Existe correlación entre la masa trofoblástica y la incidencia de hipertensión (enfermedad molar, gestación múltiple, hiperplacentosis, etc).

- La aparición de la hipertensión durante la gestación es insidiosa.

- Las pacientes primíparas tienen seis veces más probabilidad de presentar eclampsia que las multíparas, y las multíparas tienen más frecuentemente una enfermedad de base.

- La probabilidad de presentarse una hipertensión repetida en una futura gestación está relacionada con la persistencia de hipertensión más allá del décimo día postparto ( $59 \%$ vs $21 \%)$, la obesidad $(70 \%$ vs $27 \%)$, la aparición de la enfermedad antes de la semana 36 y la presencia de presión sistólica promedio de $160 \mathrm{~mm}$ de $\mathrm{Hg}(46 \%$ vs $27 \%)$.

- Las pacientes con hipertensión limítrofe (disminución de la complacencia vascular al someterse a un estímulo simpático) tienen más probabilidad de descompensa- ción y de manifestar posteriormente una hipertensión establecida.

- La presión arterial media superior o igual a $90 \mathrm{~mm}$ de $\mathrm{Hg}$ en el segundo trimestre es indicativa de hipertensión (34).

- La elevación del ácido úrico en la gestación se acompaña de desarrollo de hipertensión.

De esta forma, con los cambios hemodinámicos tempranos se inicia el proceso de "aparición de la hipertensión" (labilidad hipertensiva), que dependerá de la reserva funcional, osea, del conjunto de adaptaciones fisiológicas existentes para conservar la homeostasis. Cuando la hipertensión se hace manifiesta, en ese momento la reserva ha alcanzado tal grado de deterioro, que no es posible compensar las alteraciones hemodinámicas existentes. En este punto crítico de expresión clínica se ha consumido lo que hemos llamado el "tiempo reserva".

Mabie (40) compara el comportamiento hemodinámico de la HIE con el de pacientes hipertensas crónicas que desarrollan hipertensión inducida gestacional, encuentra que el $73 \%$ de las HIE son hiperdinámicas con elevado gasto cardíaco, al cual le corresponde una resistencia periférica normal o elevada (fig. 8). Anota que no se observan diferencias entre la forma inducida por la gestación y la forma crónica de la hipertensión y que estos hallazgos se correlacionan con la clínica de la enfermedad: taquicardia, presión de pulso aumentada, precordio hiperdinámico, soplo sistólico y extremidades calientes. Esta respuesta, refleja la incapacidad de dilatación en la circulación sistémica ante el aumento del gasto cardíaco, por lo cual podemos concluir que lo que Gant describió desde 1974 como una "pérdida de la refractariedad vascular a los cambios hemodinámicos" normales de la gestación, corresponde a una falla circulatoria que visto en otros términos, es la limitación de la reserva normal a tolerar estos cambios. En diversos estudios se ha demostrado que esta falla adaptativa ocurre muy tempranamente en la gestación (Zemel, 1990) (14).

Es tentador decir que la H.I.E (o preeclampsia) no es más que otra forma de hipertensión crónica, concepto que no se aleja de la opinión de Fishber en 1939, cuando

\section{Figura 8 \\ COMPORTAMIENTO HEMODINAMICO EN PACIENTES PREECLAMPTICAS COMPARADO CON HIPERTENSAS CRONICAS}

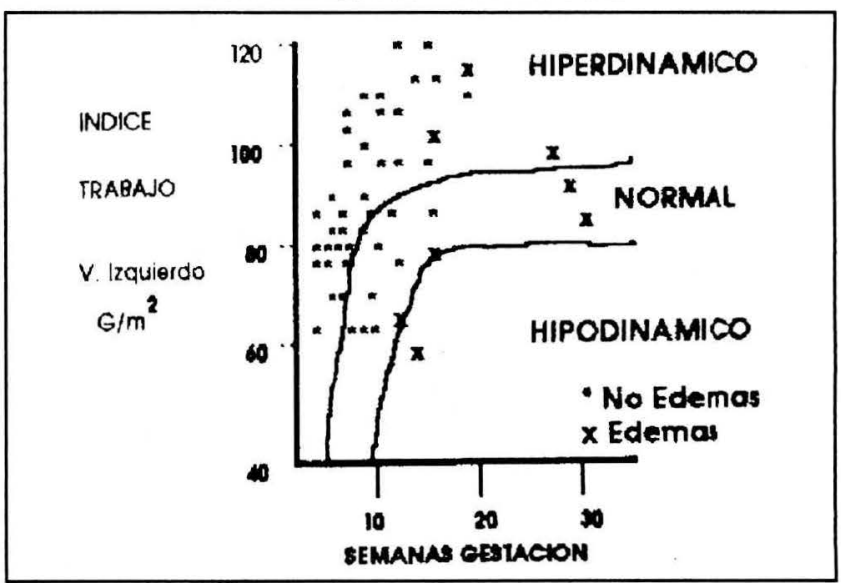


expresa el punto de vista de los internistas: “...la H.I.Eeclampsia no es una entidad sino un signo de una enfermedad hipertensiva coloreada por el embarazo".

\section{Hipótesis}

1. La preeclampsia o H.I.E. no existe como tal, es una forma de hipertensión esencial que se expresa durante la gestación como una señal de alarma de su futura aparición en el tiempo.

2. La aparición del trastorno hipertensivo depende del tiempo reserva, es decir, del momento en el cual no queda más tiempo para que la reserva funcione y conserve la idoneidad funcional de la paciente.

3. El embarazo es la sobrecarga "fisiológica" más intensa que un ser humano pueda sobrellevar, y su tolerabilidad está dependiendo directamente de la reserva que posea. Cada gestación "consume" parte de la reserva y si a ella se le agrega un fenómeno como una vasculopatía, nefropatía, etc. cada vez se hará más susceptible de desarrollar el trastorno hipertensivo.

\section{Ejemplos prácticos del tiempo reserva - sustentación}

Ya he planteado el concepto de la reserva funcional con la que se nace y que permite tolerar a través de la vida el proceso natural del envejecimiento. La presión arterial cambia sustancialmente a medida que se pierden los recursos vasodilatadores, reflejándose en la tendencia a la elevación. ¿Cuál es el tiempo reserva que posee un determinado ser humano al nacer? Inicio planteando que la reserva total es de " 1 " (uno) y que a medida que avanza en el tiempo, se reduce por el simple proceso de la vida hasta llegar a " $\mathrm{X}$ ". Al final el individuo tendrá la reserva reducida en 1-x (fig. 9) y el tiempo transcurrido para llegar allí es el tiempo-reserva, o sea el intervalo durante el cual los mecanismos homeostáticos le permiten funcionar "normal". Cuando se llega al momento "X" no quedan elementos fisiológicos de respuesta a situaciones de demanda, lo que hace aparente el deterioro funcional, aparece la enfermedad. En el control de presión arterial, la tendencia a la elevación de la tensión significa que existe ya un proceso de deterioro, cuando sobrepasa los valores normales ya no existen elementos para controlar la alteración. Los elementos de regulación funcional ya revisados, en mayor o menor medida interactúan en el control de la perfusión en respuesta a modificaciones del ambiente interno, y son determinados en forma global por la carga genética, es decir la herencia.

Para ejemplificar mejor la situación del tiempo-reserva, haré una homología con un ejemplo práctico; imagínese un país que piensa iniciar un conflicto armado, para el cual los estrategas militares planean cumplirlo en un tiempo límite, poseyendo el armamento y el ejército necesarios para "triunfar". Deben disponer además de los recursos internos mínimos para la supervivencia del ejército, de su población y garantizarse un suministro adecuado de armamento. Todo esto es la reserva de guerra y la cantidad de reserva disponible determina el tiempo reserva (consumo). Si todo marcha bien, el consumo natural de la reserva con el avance del conflicto, permitirá lograr objetivos y al final será el vencedor, lógicamente con menos reserva que la inicial pero aún con existencias y lo más importante, una nación intacta y vencedora. Si por alguna razón, inicia el conflicto con reserva de guerras menores de las necesarias, lógicamente tendrá menos tiempo para lograr el objetivo (menor tiempo reserva) o si inicia con una determinada reserva, pero durante el conflicto, es bloqueado internacionalmente y se impide la recuperación de los recursos, éstos serán consumidos antes de lo planeado, concluyendo en una franca derrota.

Veamos ahora desde el punto de vista clínico la gestación y la presencia de hipertensión:

1. El período de gestación, puede verse como un segmento corto en la evolución de la normal declinación de la reserva funcional de la vida, período durante el cual todo evento que ocurra, influirá en mayor o menor medida sobre la reserva final (fig. 10).

2. La curva de evolución de los diferentes cambios gestacionales se comporta dentro de los límites fisiológicos de la tolerancia, pudiendo llegar hasta la línea de reserva sin sobrepasarla cuando la gestación es normal.

3. En la situación de la primípara, es posible que genéticamente la paciente posea una determinación normal de la reserva, pero por la carga inmunológica

Figura 9

PROPUESTA DEL COMPORTAMIENTO DE LA

RESERVA FUNCIONAL DESDE EL NACIMIENTO

HASTA LA MUERTE, RELACION CON LA

MORBILIDAD Y CON LAS POSIBLES

SOBRECARGAS QUE SE IMPONEN EN EL CURSO DE LA VIDA

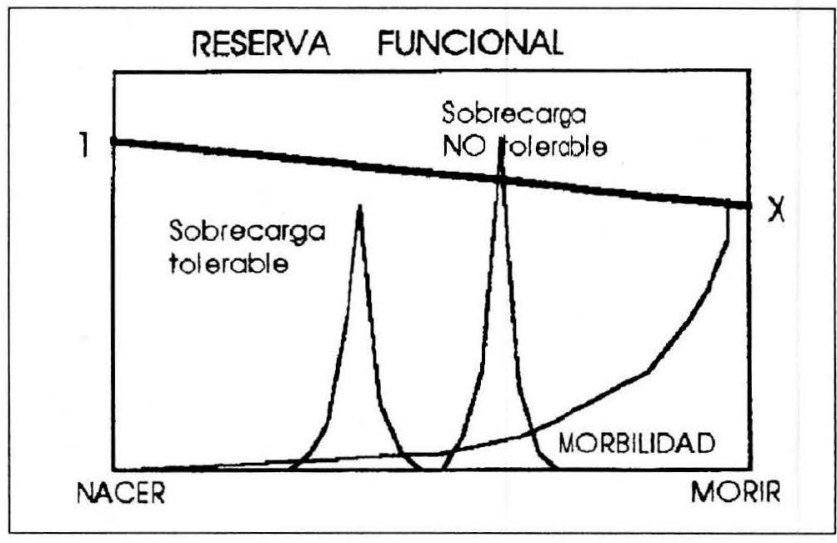

Figura 10

COMPORTAMIENTO DE LA RESERVA DURANTE EL PERIODO DE GESTACION Y SU RELACION CON LAS MODIFICACIONES ADAPTATIVAS

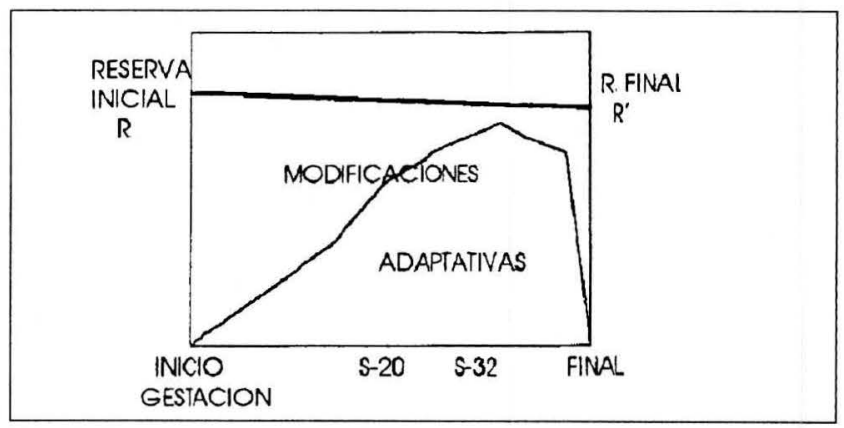


que significa el embarazo, la reserva se baje aproximando el límite de la tolerancia hacia la curva de elevación de las modificaciones gestacionales, momento en el cual se ponen en alerta los mecanismos reguladores y se manifiesta la descompensación, aparece la enfermedad. El tiempo reserva depende entonces de la carga impuesta y también de la reserva que posea cuando inicia la enfermedad. Una vez finalizado el evento, la reserva puede retomar su curso evolutivo o quedar por debajo de lo normal (fig. 11).

4. Cuando durante la gestación se presenta una noxa, por ejemplo una pielonefritis, esta sobrecarga pone a prueba la reserva inicial, bajando la línea de tolerancia hacia la curva de sobrecarga funcional apareciendo la enfermedad clínicamente. El grado de sobrecarga que ponga la noxa puede deteriorar la evolución de la reserva (fig. 12).

5. En condiciones de sobrecarga funcional como suele ocurrir en los estados de hiperplacentosis (embarazo gemelar, macrosomía, isoinmunización y en la mola), la sobrecarga suele poner a prueba la línea de tolerancia de la reserva, manifestando la alteración clínica antes de lo esperado fisiológicamente, más aún si existe una limitada reserva al iniciar el evento obstétrico (fig. 13).

6. Si al ingresar a la gestación se posee una reserva disminuida, bien sea por la determinación genética, por alteración previa o por concomitancia con otras situaciones que la disminuyan, la evolución normal

Figura 11

EFECTOS DEL FENOMENO INMUNOLOGICO PRESENTE EN LA PRIMERA GESTACION, BIEN SEA POR DISMINUCION DE LA RESERVA O POR SOBRECARGA ADAPTATIVA TEMPRANA

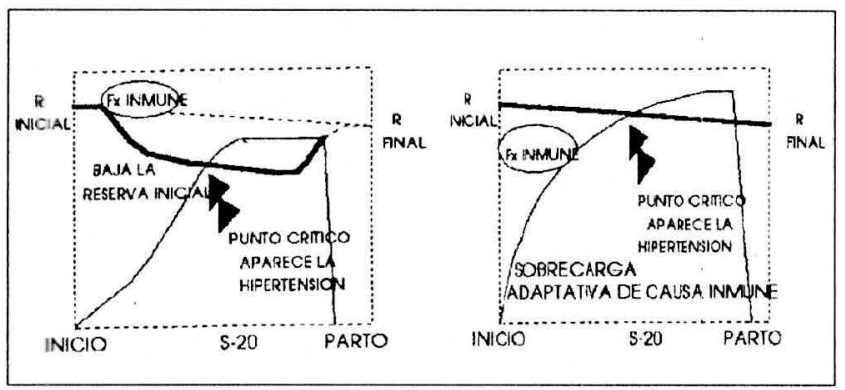

Figura 12

EFECTO DE NOXAS SOBRE LA RESERVA FUNCIONAL CON DISMINUCION DEL TIEMPO RESERVA

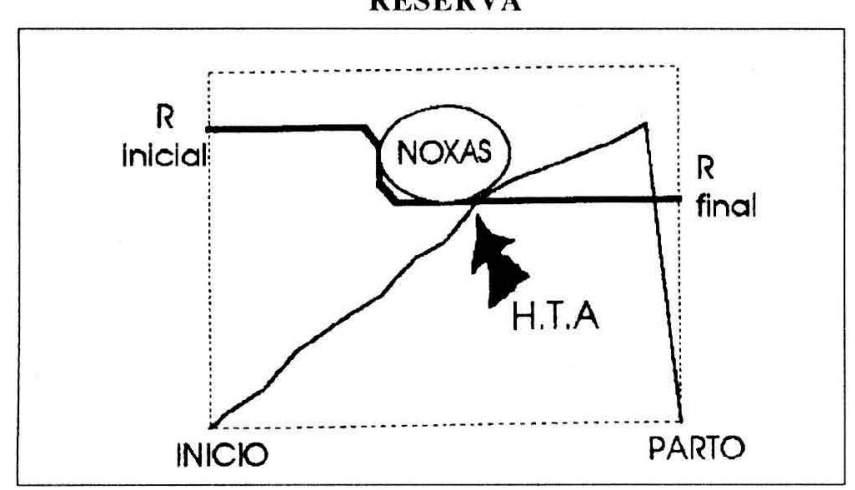

Figura 13

SOBRECARGA FUNCIONAL AUMENTADA SECUNDARIA A PROCESO DE MODIFICACION GESTACIONAL, QUE ALCANZAN A SOBREPASAR LA LINEA DE RESERVA ACORTANDO EL TIEMPO RESERVA

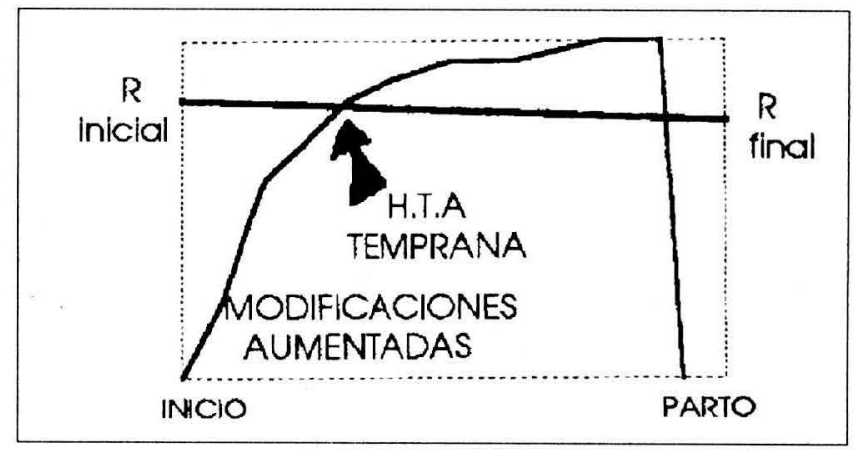

de la sobrecarga fisiológica gestacional llegará más precozmente a sobrepasar la línea de reserva, descompensando el estado clínico y apareciendo la enfermedad. En esta condición, se incluyen aquellas pacientes con hipertensión arterial previa, llamada crónica, las pacientes con vasculopatías, o nefropatías, que por su baja reserva se descompensan con los cambios normales de la gestación. En tales condiciones, se podría predecir más tempranamente la señal de alerta (factor de riesgo) (fig. 14).

Figura 14

RELACION DE LA RESERVA DISMINUIDA CON PROCESOS ADAPTATIVOS NORMALES QUE PONEN EN EVIDENCIA LA HIPERTENSION

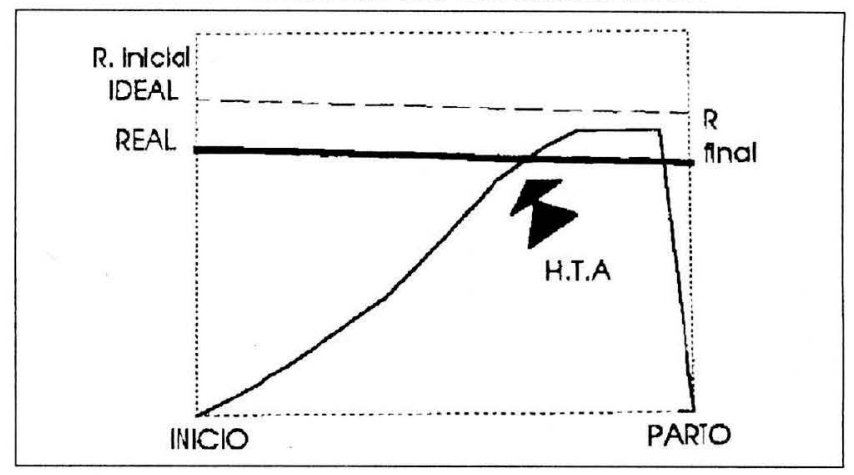

Figura 15

EFECTO DEL DETERIORO PROGRESIVO DE LA RESERVA CON RELACION A EMBARAZOS O NOXAS AGREGADAS EN EL CURSO DE LA VIDA

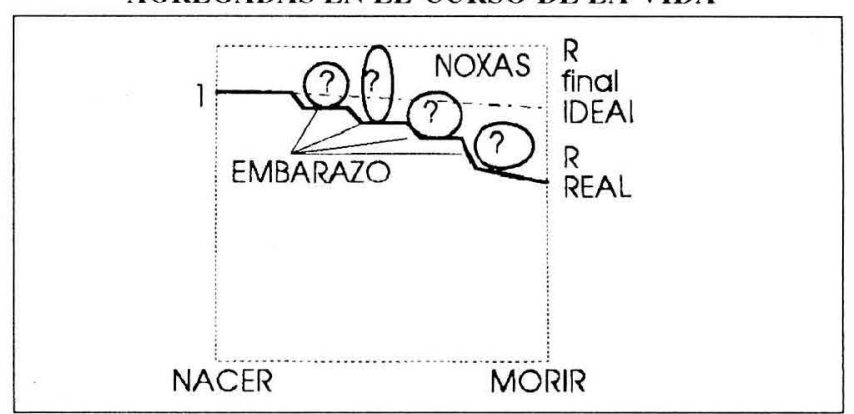


7. En definitiva, cada gestación es una prueba de esfuerzo para la reserva funcional con progresivo deterioro de ésta. Las modificaciones gestacionales se acercan cada vez más a la línea de tolerancia, con lo cual se precipita la aparición de la enfermedad hipertensiva.
En esta población debe tenerse en cuenta cada uno de los fenómenos anteriores, que se hacen más frecuentes con el aumento de la edad y con el número de gestaciones (fig. 15).

\section{BIBLIOGRAFIA}

1. Baxter JD., Perloff D., Hsueh W., Biglieri EG. The Endocrinology of Hypertension. In: Felig P., Baxter JD., Broadus AE and Frohman LA (eds.). Endocrinology and Metabolism. New York, McGraw. 1987; 693-788.

2. Williams GH and Braunwald E. Hypertensive Vascular Disease. In: Braunwald E., Isselbacher KJ., Petersdorf RG et al. (eds.). Harrison's Principles of Internal Medicine. 11th ed, New York, McGraw 1987; 1024-1037.

3. Roberts JM. Pregnancy Related Hypertension. In: Creasy RK and Resnik R. Maternal Fetal Medicine: Principles and Practice. 3th ed, Philadelphia, Saunders. 1993; 804-843.

4. Arias F. High-Risk Pregnancy and Delivery. St. Louis. Mosby. 1984; 91-120.

5. Smith LL and Thier S. Fisiopatología. Principios Biológicos de la Enfermedad. Buenos Aires, Panamericana. 1983; 913-920.

6. O'Brien WF. Predicting Preeclampsia. Obstet. Gynecol. 1990; 75: 445-452.

7. Gant NF. Embarazo e Hipertensión. In: Semin. Perinat. México, Interamericana. 1980; 175.

8. Guyton AC. Tratado de Fisiología Médica. México, Interamericana. 1984; 264-293.

9. Carretero OA and Scicli G. Local Hormonal Factors (Intracrine, Autocrine and Paracrine) in Hypertension. Hypertension 18 (Suppl 1): 1991; 58-69.

10. Pinto A., Sorrentino R., Sorrentino P et al. Endothelial Derived Relaxing Factor Released by Endothelial cells of Human Umbilical Vesseis and its Impairment in Pregnancy Induced-Hypertension Am. J. Obstet. Gynecol. 1991; 164: 507-513.

11. Panta JA., Quyyumi AA., Brush JE and Epstein SE. Normal Endothelium Dependent Vascular Relaxation in Patients with Essential Hypertension. N. Engl. J Med. 1990; 323: 22-27.

12. Belizan JM., Villar J., González L et al. Calcium Supplementation to Prevent Hypertensive Disorders of Pregnancy. N. Engl. J. Med. 1991; 325: 1399-1405.

13. Rodríguez MH., Masaki I., Mestman $\mathbf{J}$ et al. Calcium-Creatinine Ratio and Microalbuminuria in the Prediction of Preeclampsia. Am. J. Obstet. Gynecol.1988; 159: 1452-1455.

14. Zemel MB., Zemel PC., Berry S et al. Altered Platelet Calcium Metabolism as an Early Predictor of Increased Peripheral vascular Resistance and Preeclampsia in Urban Black Women. N. Engl. J. Med. 1990; 323: 434-438.

15. Parfrey NS., Wright P., Goodwin FJ et al. Blood Pressures and Hormonal Changes Following Alteration in Dietary Sodium and Potassium in Mild Essential Hypertension. Lancet 1981; 1: 59.

16. Sibai BM. Magnesium Sulfate is the Ideal Anticonvulsant in Preeclampsia-Eclampsia. Am. J. Obstet. Gynecol. 1990; 162: 11411145.

17. Iseri LI and French JH. Magnesium Nature Physiologic Calcium Blocker. Am Heart J. 1984; 108: 188-193.

18. Oates JA., Fitzgerald GA., Branch RA. Clinical implications of Prostaglandins and Thromboxane $\mathrm{A}_{2}$ Formation (part two). N Engl. J. Med. 1988; 319: 761-767.

19. Gómez P., JA. Enfermedad Hipertensiva del Embarazo. Toxemia gravídica, gestosis. Barcelona, Salvat. 1983; 236.

20. Zuspan FP. Hipertensión y Enfermedad Renal en el Embarazo. In: Clin. Obstet. Ginecol. México, Interamericana 1984; 1021-1156.

21. Brosens I., Robertson W and Dixon H. The Role of the Spiral Arteries in the Pathogenesis of the Preeclampsia. Obstet. Gynecol. Annual. N.York, Appleton. 1972; 177.

22. Williams GH and Moore TJ. The Renin-Angiotensin-Aldosterone Axis. In: Tulchinsky D and Ryan KJ. Maternal Fetal Endocrinology. Philadelphia. Saunders. 1980; 84-96.
23. Cherry SH., Berkowitz RL and Kase NG. Rovinsky and Guttmacheter's Medical, Surgical, and Gynecologic Complications of Pregnancy. Baltimore, Williams \& Wilkins. 1985; 354-375.

24. Ottoson UB., Carlstrom K., Bamber JE et al. Serum Levels of Progesterone and Some it Metabolites Including Deoxycorticosterone after Oral and Parenteral Administration. Br. J. Obstet. Gynecol. 1984; 91: 1111.

25. Parker CR., Everett RB., Whalley PJ et al. Hormone Production During Pregnancy in the Primigravid Patient. II-Plasma Levels of DOCA Throghout Pregnancy of Normal Women and Women who Developed Pregnancy-Induced Hypertension. Am. J. Obstet. Gynecol. 1980; 138: 626-631.

26. Everett RB., Worley RJ., McDonald PC et al. Modification of Vascular Responsiveness to Angiotensin II in Pregnant Women by Intravenously Infused 5-alfa Dihydroprogesterone. Am. J. Obstet. Gynecol. 1978; 131: 352-357.

27. Gant NE., Chand S., Whalley PJ et al. The Nature of Pressor Responsiveness to Angiotensin II In Humạn Pregnancy. Obstet. Gynecol. 1974; 43: 854-860.

28. Wang Y., Kay HH and Killam Ap. Decreased levels of Polyunsaturated Fatty Acids in Preeclampsia. Am. J. Obstet. Gynecol. 1991; 164: 812 818.

29. O'Brien WF., Knuppel RA., Saba HI et al. Serum Prostacyclin Binding and Half Life in Normal and Hypertensive Pregnant Women. Obstet. Gynecol. 1989; 73: 43-46.

30. Walsh SW and Coulter S. Increased Placental Progesterone may Caused ecreased Placental Prostacyclin Production in Preeclampsia. Am. J. Obstet. Gynecol. 1989; 161: 1586-1592.

31. Friedman SA. Preeclampsia: A Review of the Role of Prostaglandins. Obstet. Gynecol. 1988; 71: 122-137.

32. Hatch R. Hirsutism: Implications, Etiology and Managemnet. Obstet. Gynecol. 1981; 140: 815-820.

33. Oates JA., Fitzgerald GA., Branch RA et al. Clinical Implications of Prostaglandins and Thromboxane $A_{2}$ N. Engl. J. Med. 1988; 319: 689-698.

34. Hubel CA., Roberts JM., Taylor RN et al. Lipid Peroxidation in Pregnancy: New Perspectives on Preeclampsia Am. J. Obstet. Gynecol. 1989; 161: 1025-1034.

35. Lockwood CJ and Peters JH. Increased Plasma Levels of ED-1 Cellular Fibronectin Precede the Clinical Signs of Preeclampsia. Am. J. Obstet. Gynecol. 1990; 162: 358-362.

36. Behrman HR and Caldwell BV. Prostaglandins, Thromboxanes and Leukotrienes. In: Yen, SSC and Jaffe RB. Reproductive Endocrinology. Physiology, Pathophysiology and Clinical Management. Philadelphia, Saunders. 1986; 154-176.

37. Page EW and Christianson R. The Impact of Mean Arterial Pressure in the Second Trimester upon the Outcome of Pregnancy. Am. J. Obstet. Gynecol. 1976; 125: 740-746.

38. Capeless El and Clapp JF. Cardiovascular Changes in Early Phase of Pregnancy. Am. J. Obstet. Gynecol. 1989; 161: 1449-1453.

39. Chesley LC and Cooper DW. Genetics of Hypertension in Pregnancy. Possible Single Gene Control of Preeclampsia and Eclampsia in the descendents of Eclamptic Women. Br. J. Obstet. Gynecol. 1986; 93: 898-908.

40. Mabie WC., Ratts TE and Sibai BM. The Central Hemodynamics of Severe Preeclampsia. Am. J. Obstet. Gynecol. 1989; 161: 1443-1448.

41. Moncada S and Higgs A. The L-Arginine nitric oxid pathway. N Engl J Med 1993; 329(27): 2002-2012. 